\title{
Identical Particle Correlations in STAR
}

\author{
Z. Chajęcki ${ }^{\mathrm{a}}$ (for the STAR* Collaboration) \\ aThe Ohio State University, 191 W. Woodruff Avenue, Columbus, Ohio 43210, USA
}

Preliminary results of identical-particle correlations probing the geometric substructure of the particle-emitting source at RHIC are presented. An $m_{T}$-independent scaling of pion $\mathrm{HBT}$ radii from large (central $\mathrm{Au}+\mathrm{Au})$ to small $(\mathrm{p}+\mathrm{p})$ collision systems naively suggests comparable flow strength in all of them. Multidimensional correlation functions are studied in detail using a spherical decomposition method. In the light systems, the presence of significant long-range non-femtoscopic correlations complicates the extraction of HBT radii.

\section{Introduction}

Particle interferometry is a useful technique that provides information on the spacetime properties of nuclear matter created in high energy collisions (for the latest review articles see [1, 2] ). In this paper results of particle correlations in $\mathrm{p}+\mathrm{p}, \mathrm{d}+\mathrm{Au}$ and $\mathrm{Cu}+\mathrm{Cu}$ collisions at $\sqrt{s_{N N}}=200 \mathrm{GeV}$ and $\mathrm{Au}+\mathrm{Au}$ at $\sqrt{s_{N N}}=200$ and $62 \mathrm{GeV}$ registered by the STAR experiment are presented. Rich data statistics and the wide acceptance of the detectors gives an opportunity to do a multidimensional femtoscopic analysis. The main focus of this article is on the transverse mass $\left(m_{T}=\sqrt{k_{T}^{2}+m_{\pi}^{2}}\right)$ dependence of the HBT radii for different system sizes. This allows the study of the dynamics of the source and the conditions of the collision. Importantly, direct comparison between the $m_{T}$ dependence of radii from $\mathrm{p}+\mathrm{p}$ and $\mathrm{A}+\mathrm{A}$ collisions is possible for the first time.

\section{Multiplicity Scaling and Expansion}

Figure 1 presents the HBT radii dependence on $\left(d N_{c h} / d \eta\right)^{1 / 3}\left(d N_{c h}\right.$ - number of charged particles) for different colliding systems at different energies of the collisions. The motivation for studying such a relation is its connection to the final state geometry through the particle density at freeze-out. All STAR results, from $\mathrm{p}+\mathrm{p}, \mathrm{d}+\mathrm{Au}, \mathrm{Cu}+\mathrm{Cu}$ and $\mathrm{Au}+\mathrm{Au}$ collisions, are combined on the left panel of this figure and, as seen, all radii exhibit a scaling with $\left(d N_{c h} / d \eta\right)^{1 / 3}$. On the right panel STAR radii, this time for different range of $k_{T}$, are plotted together with AGS/SPS/RHIC systematics [1]. It is impressive that the radius parameters $R_{\text {side }}$ and $R_{\text {long }}$ follow the same curve for different collisions over a wide range of energies and, as it was checked, this observation is valid for all $k_{T}$ bins studied by STAR. It is a clear signature that the multiplicity is a scaling variable that

\footnotetext{
*For the full list of STAR authors and acknowledgments, see appendix 'Collaborations' of this volume.
} 


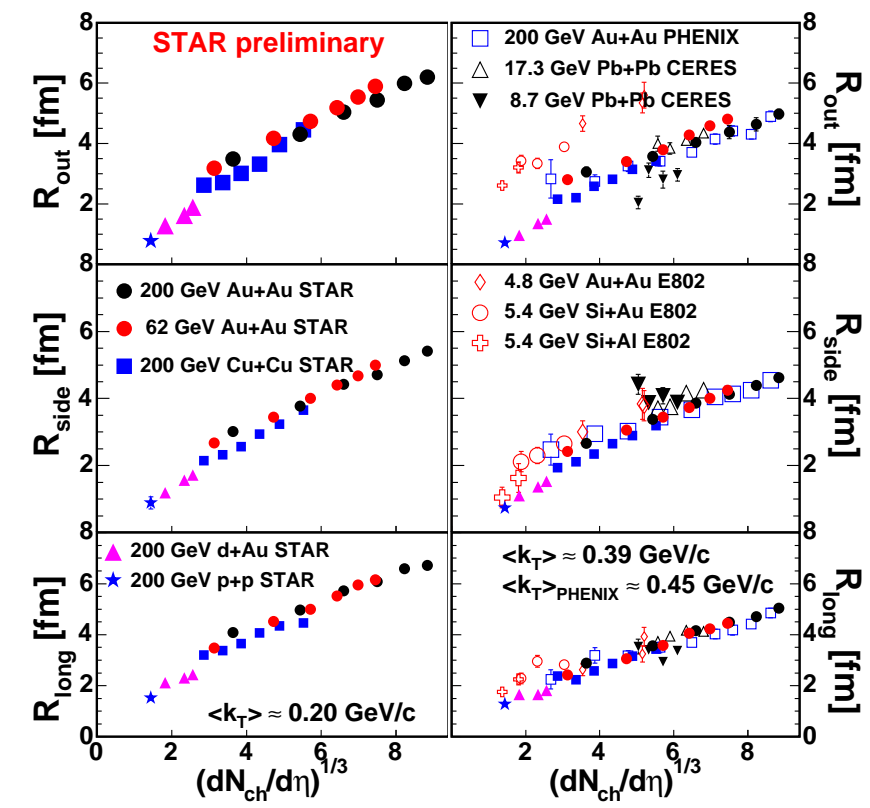

Figure 1. Femtoscopic radii dependence on the number of charged particle.

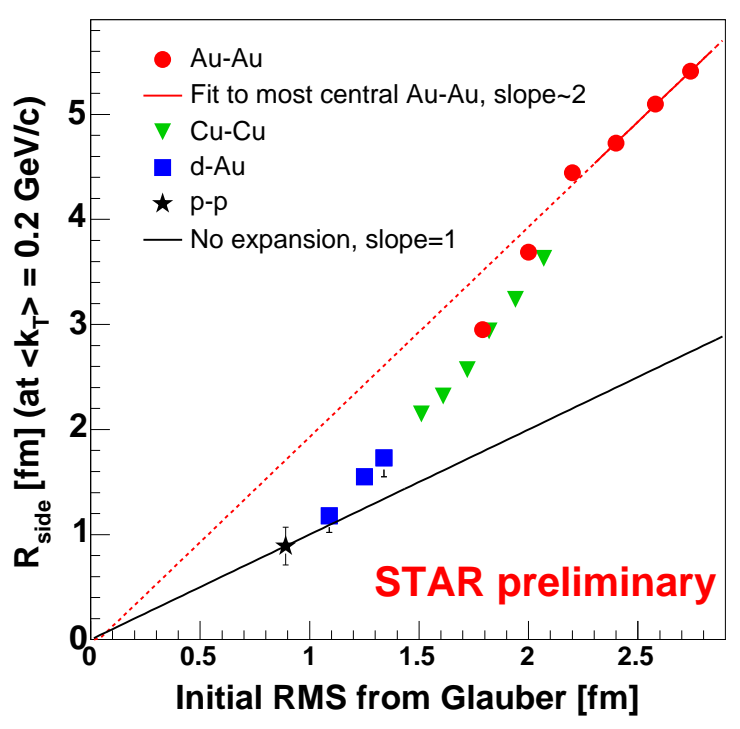

Figure 2. Final size of the source vs. initial radii calculated from Glauber model. STAR $\mathrm{Au}+\mathrm{Au}$ data from [8].

drives these HBT radius parameters. $R_{\text {out }}$ mixes space and time information. Therefore it is unclear whether to expect a simple scaling with the final state geometry.

Despite the approximately linear dependence of $R_{\text {side }}$ and $R_{\text {long }}$ on $d\left(d N_{c h} / d \eta\right)^{1 / 3}$, because of the finite intercepts of the linear relationships [ 1, 3], the data do not imply freeze-out at a constant density. The fact that the radii scale at all (linearly or not) indicates that the sideward and longitudinal radius is determined only by multiplicity, independent of energy, colliding system, or impact parameter [4, 5, 1]. This scaling, however, breaks down at much lower energies, when baryons constitute a significant fraction of the freeze-out system [4, 6, 1].

Figure 2 suggests that the relationship between initial and final geometry is not trivial, however. There, the final RMS of the source, estimated by $R_{\text {side }}$ at low $k_{T}$ [ [7], is plotted versus the initial RMS of the overlap region, estimated with a Glauber calculation. The system generated in central $\mathrm{Au}+\mathrm{Au}$ collisions undergoes a two-fold expansion, while those from the most peripheral $\mathrm{d}+\mathrm{Au}$ and $\mathrm{p}+\mathrm{p}$ collisions expand little. Does this imply that small systems are less explosive than large ones? This is explored in the next Section.

\section{Transverse Mass Dependence of HBT Radii : $p+p$ vs $\mathrm{Au}+\mathrm{Au}$}

The $m_{T}$ dependence of the femtoscopic radii in heavy ion collisions is usually attributed to collective flow of a bulk system, and recent STAR results for central $\mathrm{Au}+\mathrm{Au}$ collisions support this picture [ 8 . In a flow scenario, an approximately "universal" $m_{T}$ dependence should apply to all particle types. This is in fact observed in Figure 3 in which onedimensional radii from pion [9], charged kaon [9], neutral kaon [10, proton and antiproton [11], and proton- $\Lambda$ [12] correlations are plotted. Correlations between particles with very different masses also show characteristic signals of collective flow [13]. 


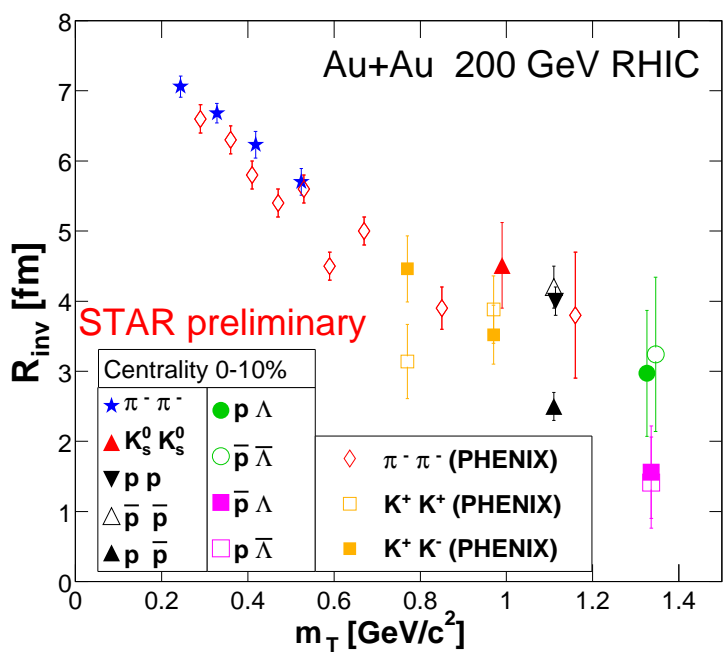

Figure 3. $m_{T}$ dependence of $R_{\text {inv }}$ for different particles. PHENIX data from [ 9$]$.

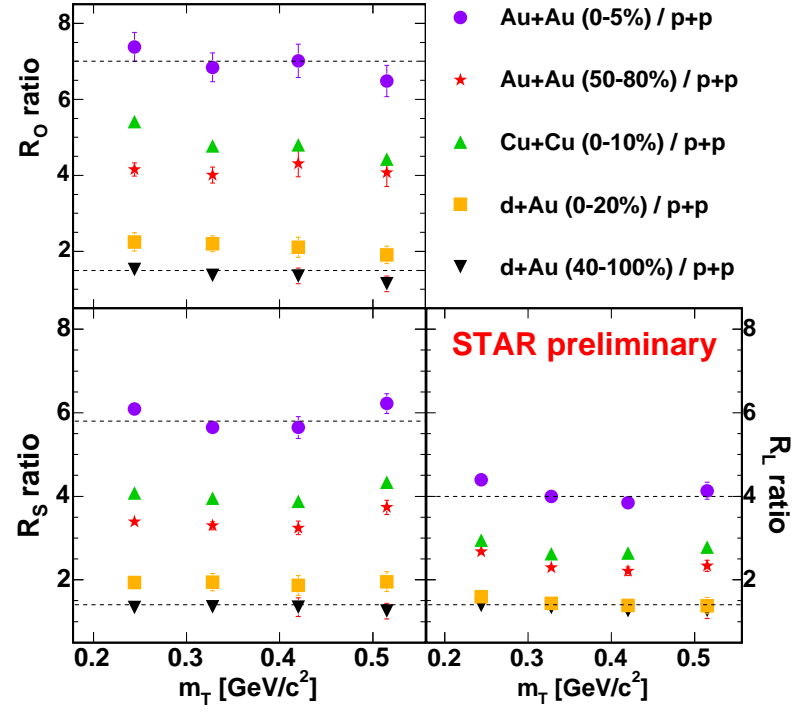

Figure 4. Ratio of $\mathrm{HBT}$ radii from $\mathrm{Au}+\mathrm{Au}$, $\mathrm{Cu}+\mathrm{Cu}$ and $\mathrm{d}+\mathrm{Au}$ by $\mathrm{p}+\mathrm{p}$ collisions at $\sqrt{s_{N N}}=200 \mathrm{GeV}$.

Decreasing $m_{T}$ dependences of the radii have previously been reported for hadron [14] and $e^{+}+e^{-}$[15] collisions. Until the STAR analysis, a direct comparison of the $m_{T}$ systematic from hadron collisions with those from $\mathrm{A}+\mathrm{A}$ collisions had been hampered by the use of quite different parameterizations in the different experiments. As shown in Figure 4, the ratio of HBT radii from $\mathrm{Au}+\mathrm{Au}$ collisions with those from $\mathrm{p}+\mathrm{p}$ collisions are approximately independent of $m_{T}$. It might be that proposed explanations for the $m_{T}$ dependence in small systems, including Heisenberg-type relations [15] and inside-outside cascade scenarios [ 16], replicate the $m_{T}$ dependence from bulk collective flow. This would be a surprising and unfortunate coincidence, suggesting that dynamical signals in the space-time sector cannot distinguish very different underlying physics. Alternatively, as proposed by Csörgö et al. [17], it could be that $\mathrm{p}+\mathrm{p}$ collisions generate a thermalized bulk system, similar to that created in heavy ion collisions. Further theoretical work on this important question would be most welcome. However, as it is mentioned in the next Section, further work is also required on the experimental side.

\section{The effect of non-femtoscopic correlations in low-multiplicity events.}

HBT radii may arise from Gaussian fits to the three-dimensional correlation function [ 1. But for low-multiplicity systems, unaccounted-for structures in the correlation function itself are observed. In particular, the value of the correlation function $C(\vec{q})$ for large $|\vec{q}|$ (larger than the scale of quantum statistics or Coulomb interactions) does not approach a common $\vec{q}$-independent value. Thus, non-femtoscopic correlations (e.g. due to momentum conservation) are becoming significant here; they have been also observed earlier, in elementary particle collisions, see e.g. [14].

The spherical decomposition of the correlation function in $\vec{q}$-space has been proposed [ 18. as a sensitive measure of long-range non-femtoscopic correlations. The lowest- $l$ com- 
ponents $A_{l, m}$ of this procedure are shown in Figure 5 . For all correlations of femtoscopic origin (Gaussian or not), $A_{l \neq 0, m}$ must vanish [18] for large $\vec{q}$; clearly other correlations are present in STAR data.

While various possible sources of these correlations are still being explored, it is interesting to simply account for the long-range correlations by adding ad-hoc terms to the Gaussian fits, which produce constant values of $A_{2,0}$ and $A_{2,2}$. It was found that the ratios shown in Figure 4 remain $m_{T}$-independent, though their values shift somewhat.

\section{Conclusions}

The results of pion interferometry for all energies and colliding systems at RHIC have been presented. In agreement with data at SPS and AGS, STAR indicates that the multiplicity is the
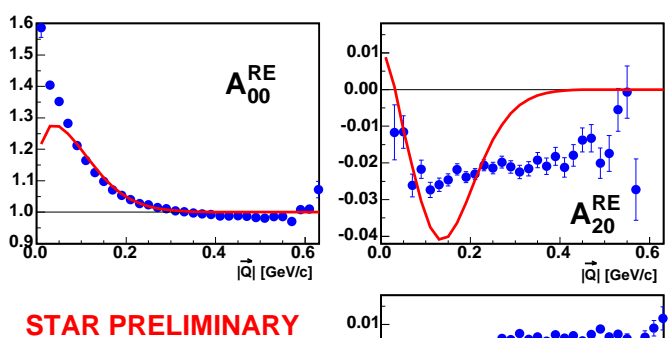

- peripheral d+Au@ $200 \mathrm{GeV}$



$k_{\mathrm{T}}:[0.15,0.60] \mathrm{GeV} / \mathrm{c}$



Figure 5: First three non-vanished components of the spherical harmonic decomposition of the correlation function for peripheral $\mathrm{d}+\mathrm{Au}$ collisions. scaling variable that determines the size of the source at freeze-out. Perhaps surprisingly, the $m_{T}$ dependence of HBT radii appears to be independent of collision species or multiplicity. Finally, a problem with the baseline of the correlation function for low multiplicity collisions has been reported, and a promising tool based on the spherical harmonic decomposition of the correlation function has been used in order to address it. The physics of this structure remains under investigation.

\section{REFERENCES}

1. M. Lisa, S. Pratt, R. Solz, U. Wiedemann, ArXiv:nucl-ex/0505014

2. R. Lednický, these proceedings, ArXiv:nulc-th/0510020 T. Csörgö, ArXiv:nucl-th/0505019, S.S. Padula, ArXiv:nucl-th/0412103.

3. S.S. Adler et al. (PHENIX Collaboration), Phys. Rev. Lett. 93 (2004) 152302.

4. R. Stock, Annalen der Physik, 48 (1991) 195.

5. T. Csörgó and L. P. Csernai, Phys. Lett. B 333 (1994) 494.

6. D. Adamov́a et al. (CERES Collaboration), Phys. Rev. Lett. 90 (2003) 022301.

7. F. Retiere, M. A. Lisa, Phys. Rev. C 70 (2004) 044907.

8. J. Adams et al. (STAR Collaboration), Phys. Rev. C 71 (2005) 044906.

9. M. Heffner (for the PHENIX Collaboration), J. Phys. G 30 (2004) S1043-S1047.

10. S. Bekele (for the STAR Collaboration), J. Phys. G 30 (2004) S229-S234.

11. H. Gos, QM 2005 Poster Presentation, to appear in Nukleonika.

12. P. Chaloupka, these proceedings, ArXiv:nucl-ex/0510064.

13. J. Adams et al. (STAR Collaboration), Phys. Rev. Lett. 91 (2003) 262302.

14. N.M. Agababyan et al. (NA22 Collaboration) Z. Phys. C59 (1993) 195.

15. G. Alexander, ArXiv:hep-ph/0302130.

16. A. Bialas and K. Zalewski, Acta Phys. Polon. B 30 (1999) 359.

17. T. Csörgö, M. Csanád, B. Lörstad, A. Ster, ArXiv:hep-ph/0406042.

18. Z. Chajęcki et al. (for the STAR Collaboration), ArXiv:nucl-ex/0505009. 